\title{
Efficient organometallic spin filter between single-wall carbon nanotube or graphene electrodes
}

Koleini, Mohammad; Paulsson, Magnus; Brandbyge, Mads

Published in:

Physical Review Letters

Link to article, DOI:

10.1103/PhysRevLett.98.197202

Publication date:

2007

Document Version

Publisher's PDF, also known as Version of record

Link back to DTU Orbit

Citation (APA):

Koleini, M., Paulsson, M., \& Brandbyge, M. (2007). Efficient organometallic spin filter between single-wall carbon nanotube or graphene electrodes. Physical Review Letters, 98(19), 197202.

https://doi.org/10.1103/PhysRevLett.98.197202

\section{General rights}

Copyright and moral rights for the publications made accessible in the public portal are retained by the authors and/or other copyright owners and it is a condition of accessing publications that users recognise and abide by the legal requirements associated with these rights.

- Users may download and print one copy of any publication from the public portal for the purpose of private study or research.

- You may not further distribute the material or use it for any profit-making activity or commercial gain

- You may freely distribute the URL identifying the publication in the public portal 


\title{
Efficient Organometallic Spin Filter between Single-Wall Carbon Nanotube or Graphene Electrodes
}

\author{
Mohammad Koleini, Magnus Paulsson, and Mads Brandbyge* \\ Department of Micro- and Nanotechnology (MIC), NanoDTU, Technical University of Denmark (DTU), \\ Ørsteds Plads, Bldg. 345E, DK-2800 Lyngby, Denmark
}

(Received 29 November 2006; published 7 May 2007)

\begin{abstract}
We present a theoretical study of spin transport in a class of molecular systems consisting of an organometallic benzene-vanadium cluster placed in between graphene or single-wall carbon-nanotube-model contacts. A $b$ initio modeling is performed by combining spin density functional theory and nonequilibrium Green's function techniques. We consider weak and strong cluster-contact bonds. Depending on the bonding we find from $73 \%$ (strong bonds) up to $99 \%$ (weak bonds) spin polarization of the electron transmission, and enhanced polarization with increased cluster length.
\end{abstract}

PACS numbers: 85.75. $-\mathrm{d}, 73.63 . \mathrm{Fg}, 75.75 .+\mathrm{a}, 85.65 .+\mathrm{h}$

Carbon nanotubes (CNTs) [1] and other graphene-based systems [2] are considered strong candidate materials for future nanoscale quantum electronic devices due to their ballistic transport characteristics. Moreover, the long spinrelaxation length in these materials hold promise for spintronics applications. There is therefore great interest in the possibility of introducing and studying spin-polarized currents in graphene-based systems.

Spin transport has been studied both experimentally [36] and theoretically [7] for CNTs in contact with magnetic electrodes. Spin polarization has also been seen in CNTs when doped with magnetic adatoms or molecules [8-10]. These results indicate that one can use CNTs as spintransport channels with ballistic transport characteristics, with the additional possibility of chemical functionalization. In general, carbon based systems seem advantageous for spin transport due to weak spin decoherence, and are therefore currently under intense investigation. In addition to CNTs, several experiments regarding spin transport through organic molecules have been done, among them are observation of spin injection and detection in $\pi$-conjugated molecules [11], spin transfer across molecular bridges [12], and spin transport in organic tunnel junctions [13]. Theoretical investigations include spin transport through 1,4-benzene-dithiolate molecules [14], spin valves based on two Cobaltocene moieties [15], and evidence of Giant Magnetoresistance (GMR) in alkane-dithiolate and phenyl-dithiolate molecules between nickel contacts [16]. Very recently theoretical studies have indicated that graphene nanoribbons may act half-metallic [17], that is, metallic and semiconducting behavior of the respective minority and majority spins, which in turn means $100 \%$ spin-polarized currents for low voltage bias.

Electrically contacted junctions can be manipulated and formed between individual nanotubes [18] or between nanotubes and graphite [19]. In this Letter we propose that an adsorbed layer of benzene-vanadium (VBZ) organometallic molecular clusters can act as a highly efficient spin filter when placed in a junction between carbon elec- trodes. The junction could, e.g., be formed first adsorbing VBZ on a nanotube or graphene electrode, which is then by manipulation contacted by a nanotube. The spin filter facilitates a highly spin-polarized current in the carbon electrodes even in the case when a weak spin polarization is introduced in one of these by a ferromagnetic contact $[5,20]$. The role of the ferromagnetic contact being here only to fix the spin of the molecule and thereby the polarization of the current. Our study is based on the ab initio pseudopotential spin-polarized density functional theory (DFT), combined with nonequilibrium Green's function transport calculations. We concentrate on the idealized situation of a single VBZ contact between two nonpolarized carbon electrodes, assuming all clusters in the junction conduct similarly; see Fig. 1. We obtain a large spin-filter effect quite independent of the detailed bonding of the cluster to the electrodes. We find from $73 \%$ (strong bonds) up to $99 \%$ (weak bonds) spin polarization of the electron transmission, with enhanced polarization for increasing cluster length.

The organometallic cluster we consider is the multidecker benzene-vanadium sandwich $\left(\mathrm{V}_{n} \mathrm{BZ}_{m}\right.$ where $\mathrm{BZ}=$ $\mathrm{C}_{6} \mathrm{H}_{6}$ and $|n-m| \leq 1$ ), which have been synthesized recently from laser-vaporized vanadium atoms and $\mathrm{BZ}$ $[21,22]$. Mass spectrometric measurements as well as theoretical studies indicate a linear geometry, see e.g. $\mathrm{V}_{4} \mathrm{BZ}_{3}$ in Fig. 1, of lengths up to $n=6(20 \AA)$, and ending with either $\mathrm{BZ}$ or $\mathrm{V}$ on each side. An integer magnetic moment of $1.00 \mu_{B}$ per VBZ unit cell has been found. Infinite chains of this cluster $(n=\infty)$ have been studied by spin-polarized DFT [23] and they show half-metallic ferromagnetic behavior with a semiconducting gap of $1.12 \mathrm{eV}$ for the majority spin, while the minority spin component is metallic. Very recently, Maslyuk et al. [24] showed that the clusters act as almost perfect spin filters when placed between magnetic contacts of nickel or cobalt.

Considering the complexity problem and in order to make the computations tractable, we neglect curvature effects in the nanotube electrodes (Fig. 1 [25]) and use 


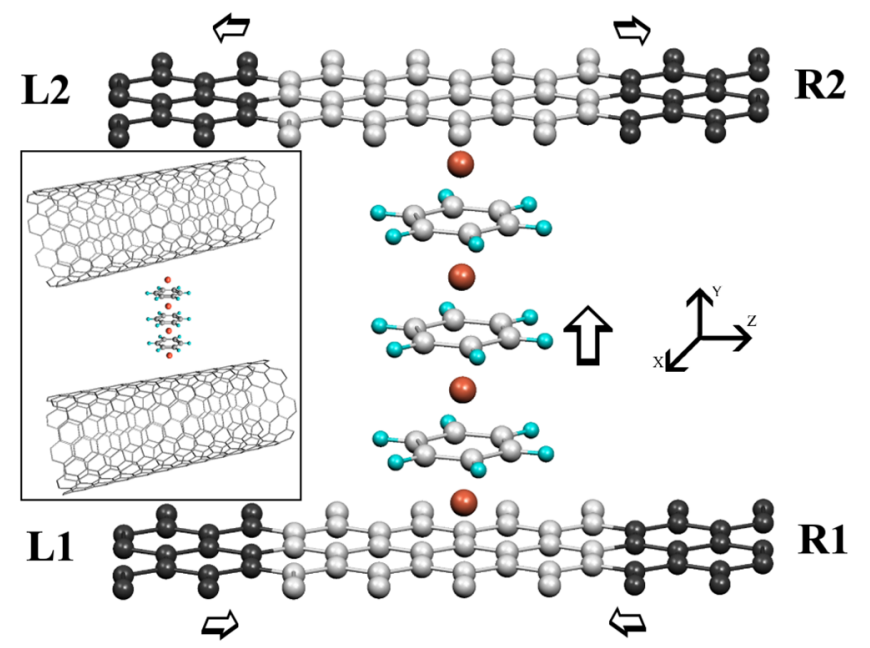

FIG. 1 (color online). The geometry with a $\mathrm{V}_{4} \mathrm{BZ}_{3}$ contacting two graphene electrodes. The arrow shows the current direction from contact " $1 "=\{L 1, R 1\}$ to contact " $2 "=\{L 2, R 2\}$. Periodic boundary conditions are employed in the $x$ direction, while transport in the graphene contacts is along $z$. The $x$-periodic contacts can also be viewed as the GSM of armchair nanotube contacts (inset).

the graphene sheet model (GSM) of metallic (armchair) carbon nanotubes [26]. We have used the ab initio pseudopotential density functional method as implemented in the SIESTA code [27] to obtain the electronic structure from spin-polarized DFT [28]. Our spin-transport calculation is based on the nonequilibrium Green's function method [30] extended to spin-polarized systems [31]. We consider here low bias and limit our calculation to transport at the Fermi energy only.

The cluster can end with either a BZ or a V and thus can bond upright to a graphene or CNT electrode with either a strong covalent bond between $\mathrm{V}$ and the electrode, or a weak van der Waals bond between BZ and the electrode. Our calculated binding energy between a single $\mathrm{V}$ adatom on a graphene sheet is $1.8 \mathrm{eV}$ with a relaxed distance of $1.7 \AA$. This strong binding is in accordance with previous studies of $\mathrm{V}$ adatoms adsorbed on CNTs [9,32]. Different orientations of $\mathrm{BZ}$ with respect to graphene are possible. We show the results for the bridge configuration, where the center of BZ is over the center of a bond in the graphene contact. This is one of the most energetically stable arrangements [33], with a DFT binding energy around $0.24 \mathrm{eV}$, and BZ-graphene distance of $3.2 \AA$ as in other $s p^{2}$-like systems. We note that other geometries ("stack" - BZ center above a graphene atom, "hexagon" with BZ above a graphene hexagon) do not change our results significantly.

The setup of the transport calculation for the geometry in Fig. 1 differs slightly from the more standard two-probe setups [30]. The GSM model that we use for contacts is equivalent to using graphene electrodes with periodic boundary conditions in the direction perpendicular to the transport, $x$ direction in Fig. 1. Our results do not change significantly when enlarging the $x$ period, i.e., tube circumference for the GSM. In this geometry, there are four current terminals. The current will enter the cluster from left and right terminals in one graphene sheet ( $L 1$ and $R 1$ ), and exit the left and right terminals in other sheet ( $L 2$ and $R 2$ ). The darker carbon atoms represent the contacting unit cells of the four semi-infinite electrodes. To solve the transport problem we consider a self-energy for each of the four electrodes $\left(\Sigma_{L 1}, \Sigma_{L 2}, \Sigma_{R 1}, \Sigma_{R 2}\right)$. The self-energy, $\boldsymbol{\Sigma}_{i}$, of each contact $i=1,2$ is composed of the two "conventional two-probe" self-energies [31] corresponding to semi-infinite extensions to the left and right,

$$
\Sigma_{1}=\left(\Sigma_{L 1}+\Sigma_{R 1}\right)
$$

and similarly for " 2 "; see Fig. 1. The transmission function for majority (minority) spin $(\sigma=\uparrow / \downarrow)$ from graphene contact 1 to graphene contact 2 is calculated using the Landauer formula,

$$
T_{\sigma}(E)=\operatorname{Tr}\left[\mathbf{G}_{D, \sigma}^{\dagger} \boldsymbol{\Gamma}_{1} \mathbf{G}_{D, \sigma} \boldsymbol{\Gamma}_{2}\right](E),
$$

where the trace runs over states in the device region consisting of the VBZ cluster, perturbed graphene parts (lightcolored part in Fig. 1), and electrode-connected graphene (black in Fig. 1). Note that $\boldsymbol{\Gamma}_{i}(E)=-2 \operatorname{Im}\left[\boldsymbol{\Sigma}_{i}(E)\right], i=1$, 2 , is nonzero only in the electrode-connected (black) parts, and are independent of spin since the semi-infinite graphene electrodes (not shown) are not polarized.

We consider the three possible classes of clusters: (I) Vanadium atoms on both end sides $\left(\mathrm{V}_{n+1} \mathrm{BZ}_{n}\right)$, (II) One vanadium and one benzene on each end side $\left(\mathrm{V}_{n} \mathrm{BZ}_{n}\right)$, and (III) benzenes on both end sides $\left(\mathrm{V}_{n} \mathrm{BZ}_{n+1}\right)$. In Fig. 2 we show the calculated spin-resolved transmission for the three classes of systems. If we, for the moment, ignore the resonant tunneling peak in Fig. 2(c), we find that the absolute magnitude of the transmission is determined by the strength of the contact-cluster coupling. The strong covalent vanadium-graphene bond, causes overall larger transmission than weak benzene-graphene bonding. The resonant transmission peak for the class III structures is possible because of the weak interaction between cluster and contact in this case which enables an almost localized state on the cluster. The position of the resonance peak is sensitive to the details of the structure, e.g., exact bonding geometry and size of cluster. However, the spin polarization of the transmission is large even out of resonance.

To quantify the results, we use the transmission spin polarization (TSP) and charge spin polarization (CSP) measures defined as,

$$
\begin{gathered}
\mathrm{TSP}=\frac{T_{\mathrm{Min}}-T_{\mathrm{Maj}}}{T_{\mathrm{Min}}+T_{\mathrm{Maj}}} \\
\mathrm{CSP}=\left(Q_{\mathrm{Maj}}-Q_{\mathrm{Min}}\right) .
\end{gathered}
$$

The values of TSP and CSP for the different systems are 


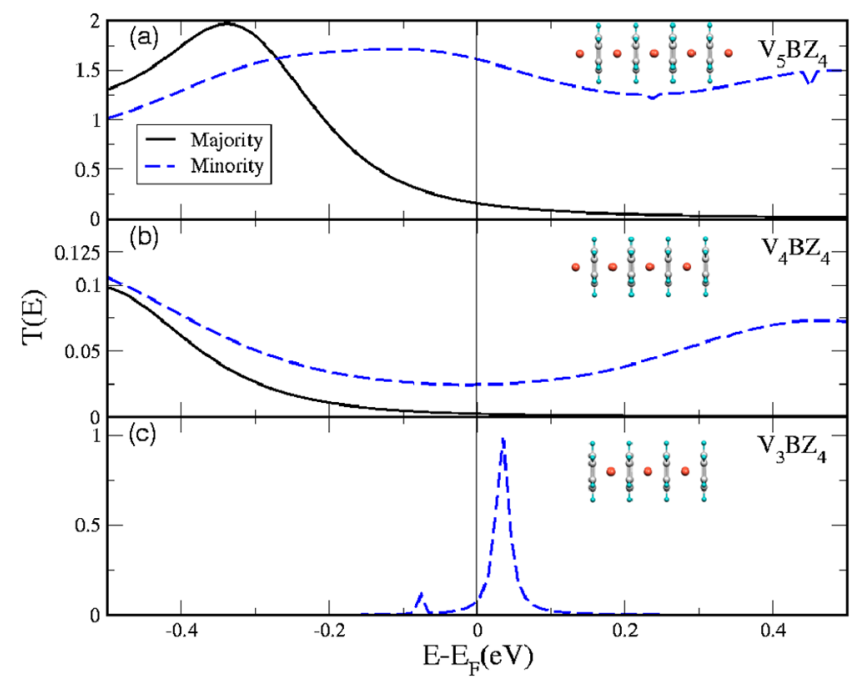

FIG. 2 (color online). Transmission functions of representatives of the three types of contacts (I-III): (a) strong V bonds to both contacts (I), (b) a strong V bond and a weak BZ-bond to the contacts (II), and (c) weak BZ-bonds to both contacts (III). In (b) and (c) the "bridge" BZ-graphene configuration was considered. Note the different scales on the transmission axes.

summarized in Table I. We see that in class III, the TSP is almost perfect [the majority spin component transmission is almost zero in Fig. 2(c)]. This extraordinary spintransport polarization is partly due to the resonance discussed above. However, for class I and II the spin-transport polarization is still above $73 \%$ which promises a very efficient spin-filter effect even for the small clusters. In each class, the CSP will increase with cluster length since the vanadium atoms couple ferromagnetically [21]. In addition, increasing the length of cluster cause the transmission of the majority spin component to decrease exponentially while the transmission for minority component is mostly unaffected by length. This will in turn also enhance the TSP with cluster length.

To ensure that the large TSP is not sensitive to the details of the geometrical arrangement of the junctions, we

TABLE I. Summary of transmission and charge spin polarization (TSP/CSP) for the different classes of molecular clusters (IIII).

\begin{tabular}{lccc}
\hline \hline & Cluster & TSP $(\%)$ & CSP \\
\hline \multirow{3}{*}{ Class I } & $\mathrm{V}_{3} \mathrm{BZ}_{2}$ & 73 & 4.9 \\
& $\mathrm{~V}_{4} \mathrm{BZ}_{3}$ & 77 & 5.8 \\
& $\mathrm{~V}_{5} \mathrm{BZ}_{4}$ & 81 & 6.8 \\
Class II & $\mathrm{V}_{3} \mathrm{BZ}_{3}$ (Bridge) & 91 & 6.6 \\
& $\mathrm{~V}_{4} \mathrm{BZ}_{4}$ (Bridge) & 80 & 7.6 \\
& $\mathrm{~V}_{5} \mathrm{BZ}_{5}$ (Bridge) & 84 & 8.3 \\
\multirow{4}{*}{ Class III } & $\mathrm{V}_{2} \mathrm{BZ}_{3}$ (Bridge-Bridge) & 93 & 2.4 \\
& $\mathrm{~V}_{3} \mathrm{BZ}_{4}$ (Bridge-Bridge) & 99 & 3.6 \\
& $\mathrm{~V}_{4} \mathrm{BZ}_{5}$ (Bridge-Bridge) & 99 & 4.7 \\
\hline \hline
\end{tabular}

changed the bond length between end $\mathrm{BZ}$ and graphene contacts by $10 \%$, as well as translating and rotating the cluster along its primary axis (perpendicular to the graphene sheets) to obtain different BZ-graphene bonding geometries, e.g., bridge, stack, or hexagon. We found that TSP changes less than $10 \%$ in the most unfavorable case.

The large and robust spin polarization in the transmission for all geometries studied here can be traced directly to the half-metallic behavior of the infinite VBZ wire, which shows $100 \%$ spin polarization at the Fermi level. It is only the minority spin bands of the infinite VBZ wire, which cross the Fermi level and cause metallic conduction. In agreement with earlier studies we find 3 crossing bands (2 degenerate) corresponding to Bloch waves with $d_{x y} /$ $d_{x^{2}-y^{2}}$ and $d_{z^{2}}$ character on the V atoms, see Fig. 3(a) and 3(b). The $d_{z^{2}}$ band display little dispersion since the $d_{z^{2}}$ are only able to couple weakly through the BZ via the hydrogen atoms. On the other hand, the degenerate $d_{x y} / d_{x^{2}-y^{2}}$ bands couple strongly through the BZ $\pi$-orbitals leading to larger dispersion. From the band structure we anticipate a maximum of 3 transport channels for the finite cluster. However, in our transport calculations of the finite cluster we find only 2 equally contributing minority spin transmission eigenchannels corresponding to scattering states with $d_{x y} / d_{x^{2}-y^{2}}$ character. The more localized $d_{z^{2}}$ states only contribute to the spin polarization and not to the transport. The scattering states of the two highest transmitting eigenchannels [34] are presented in Fig. 3(c) for $\mathrm{V}_{4} \mathrm{BZ}_{3}$ for each of the two spin components. The corresponding transmission eigenvalues are $T_{1}=0.10, T_{2}=$ 0.05 and $T_{1}=0.60, T_{2}=0.59$ for the majority and minority spin components, respectively. A decaying behavior through the cluster is observed for the majority spin wave functions which explains the increase in TSP with cluster length. We draw similar conclusions about transport channels for the other structures we have studied. The appreciable spin-filter effect found even for the strongly bound, short $\mathrm{V}_{3} \mathrm{BZ}_{2}$, is presumably due to the rather similar type of bonding of vanadium to graphene and benzene.

In summary, we have shown a high degree of spin polarization of electron transmission in a class of molecular systems consisting of organometallic half-metallic benzene-vanadium clusters sandwiched between CNT or graphene contacts. So far the majority of studies in molecular spintronics have focused on ferromagnetic contacts. In this work we have investigated another category of systems where the contacts are nonmagnetic and magnetization is solely due to the molecule. The TSP depends on the nature of bonds between cluster and contacts; however, the high values for TSP (from $73 \%$ to $99 \%$ ) in the different structures show that this effect does not depend critically on a specific structure or orientation of $\mathrm{V}_{n} \mathrm{BZ}_{m}$ cluster. We suggest these could be used to spin filter electronic current in graphene-based nanodevices when the molecular spin is fixed by external means. 

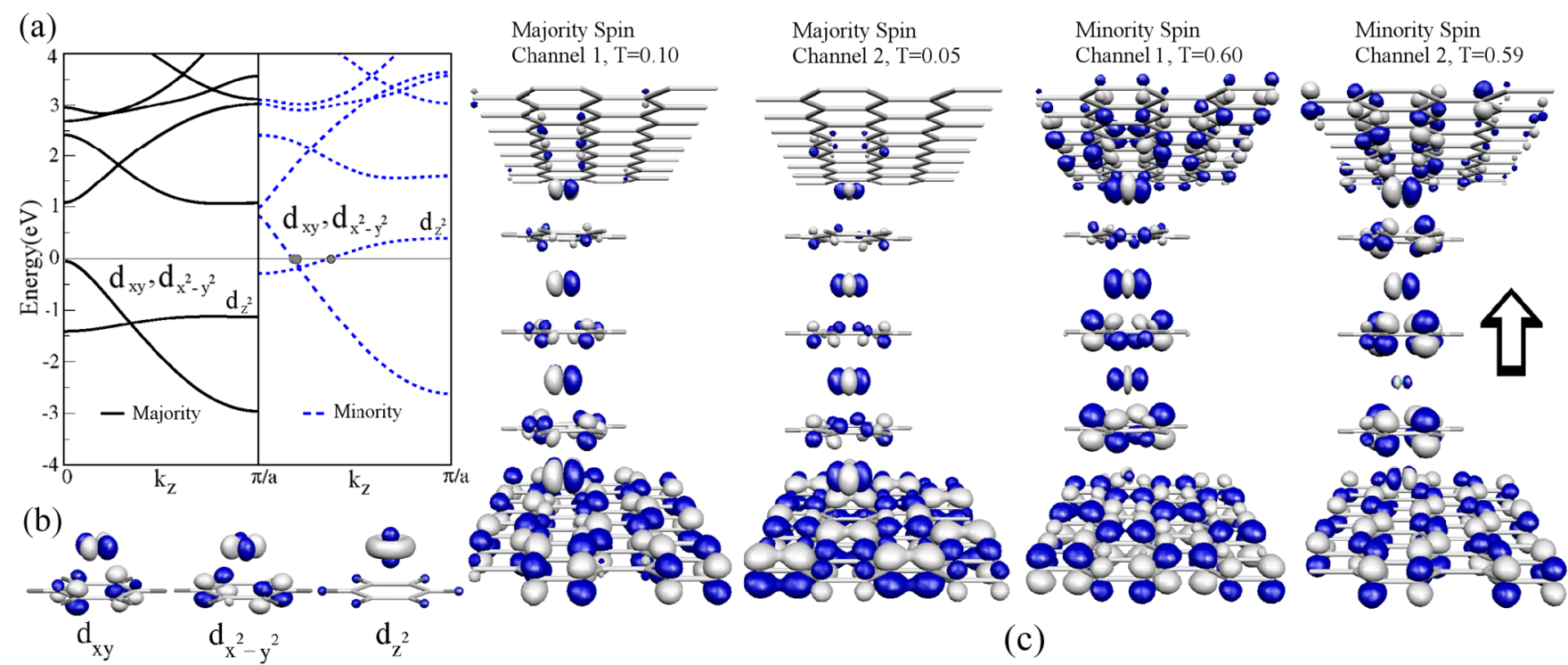
Majority Spin
Channel $1, \mathrm{~T}=0.10$
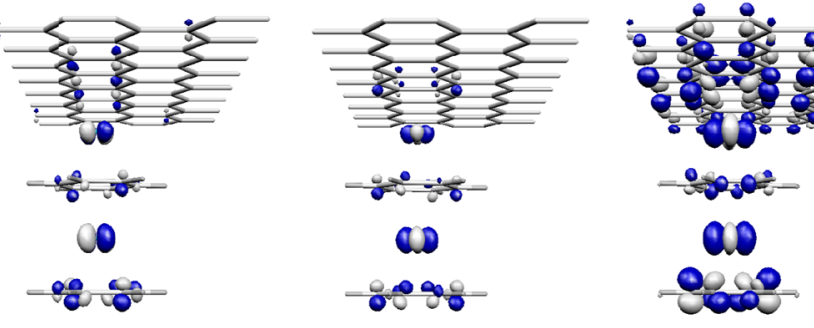

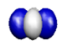
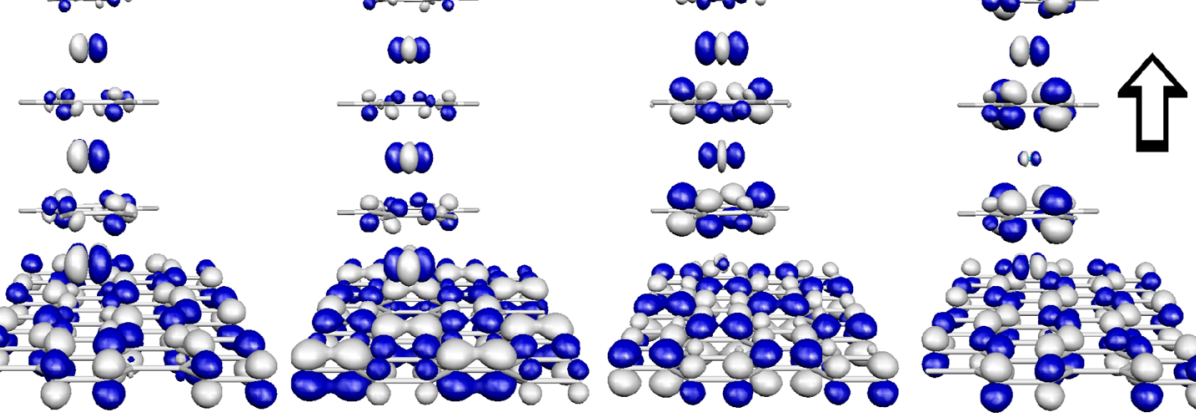

(c)

FIG. 3 (color online). (a) The spin-resolved band structure of VBZ wire. (b) The real part of the Bloch states in one VBZ unit cell. (c) Plots of the real part of the scattering states of $\mathrm{V}_{4} \mathrm{BZ}_{3}$ for two conducting channels for each spin component. The arrow shows the current direction. The system conducts the minority spin component in both channels, as can be seen from the propagating wave function from electrode 1 to 2 (bottom to top), while the wave function is decaying in the molecule and conduction is blocked for majority spin.

The authors thank V. V. Maslyuk and A.-P. Jauho for useful discussions. This work, as a part of the ESF EUROCORES (SASMEC), was supported by the SNF and the EC 6FP. Computational resources were provided by the Danish Center for Scientific Computations (DCSC).

*Electronic address: mbr@mic.dtu.dk

[1] P. Avouris, Acc. Chem. Res. 35, 1026 (2002).

[2] C. Berger et al., Science 312, 1191 (2006).

[3] K. Tsukagoshi, B. Alphenaar, and H. Ago, Nature (London) 401, 572 (1999).

[4] B. Zhao et al., Appl. Phys. Lett. 80, 3144 (2002).

[5] B. Nagabhirava et al., Appl. Phys. Lett. 88, 023503 (2006).

[6] S. Sahoo et al., Nature Phys. 1, 99 (2005).

[7] H. Mehrez, J. Taylor, H. Guo, J. Wang, and C. Roland et al., Phys. Rev. Lett. 84, 2682 (2000).

[8] V. García-Suárez, J. Ferrer, and C. Lambert, Phys. Rev. Lett. 96, 106804 (2006).

[9] C. K. Yang, J. Zhao, and J. P. Lu, Nano Lett. 4, 561 (2004).

[10] C. K. Yang, J. Zhao, and J.P. Lu, Phys. Rev. Lett. 90, 257203 (2003).

[11] Z. Xiong, Z. Di Wu, and J. Shi, Nature (London) 427, 821 (2004).

[12] M. Ouyang and D.D. Awschalom, Science 301, 1074 (2003).

[13] J. R. Petta, S. K. Slater, and D. C. Ralph, Phys. Rev. Lett. 93, 136601 (2004).

[14] E. G. Emberly and G. Kirczenow, Chem. Phys. 281, 311 (2002).

[15] R. Liu, S. Ke, H. Baranger, and W. Yang, Nano Lett. 5, 1959 (2005).
[16] A. R. Rocha et al., Nat. Mater. 4, 335 (2005).

[17] Y. W. Son, M. L. Cohen, and S. G. Louie, Nature (London) 444, 347 (2006).

[18] X. Huang et al., Nano Lett. 5, 1515 (2005).

[19] S. Paulson et al., Science 290, 1742 (2000).

[20] O. Cespedes et al., J. Phys. Condens. Matter 16, L155 (2004).

[21] K. Miyajima et al., J. Am. Chem. Soc. 126, 13202 (2004).

[22] J. Wang, P. H. Acioli, and J. Jellinek, J. Am. Chem. Soc. 127, 2812 (2005).

[23] M. Rahman, H. Kasai, and E. Dy, Jpn. J. Appl. Phys. 44, 7954 (2005).

[24] V. V. Maslyuk et al., Phys. Rev. Lett. 97, 097201 (2006).

[25] Relaxed geometries: $d_{\mathrm{VBZ}}=1.695 \AA, r_{\mathrm{CC}}=1.45 \AA$, and $r_{\mathrm{CH}}=1.09 \AA$ in accordance with [24]. We used the most stable structure with $D_{6 h}$ symmetry.

[26] C. T. White and J. W. Mintmire, J. Phys. Chem. B 109, 52 (2005).

[27] J. Soler et al., J. Phys. Condens. Matter 14, 2745 (2002).

[28] DZP basis (energy-shift $0.01 \mathrm{Ry}$ ), grid cut-off $150 \mathrm{Ry}$, GGA-PBE for exch.-corr. [29]. The GSM was tested using $10 k$ points in the periodic $x$ dir.: The $k$-averaged transmissions did not change noticeably.

[29] J. P. Perdew, K. Burke, and M. Ernzerhof, Phys. Rev. Lett. 77, 3865 (1996).

[30] M. Brandbyge et al., Phys. Rev. B 65, 165401 (2002).

[31] S. Datta, Electronic Transport in Mesoscopic Systems (Cambridge University Press, Cambridge, England, 1997).

[32] E. Durgun, S. Dag, S. Ciraci, and O. Gulseren, J. Phys. Chem. B 108, 575 (2004).

[33] F. Tournus and J.C. Charlier, Phys. Rev. B 71, 165421 (2005).

[34] M. Paulsson and M. Brandbyge, cond-mat/0702295. 